\title{
Los adultos mayores, el cuidado de los nietos y su calidad de vida
}

\section{Older adults, caring for grandchildren and their quality of life}

\author{
PONCE-CONTRERAS, María Guadalupe†*, GONZÁLEZ-ÁLVAREZ, Mireya Del Carmen, PÉREZ- \\ BRIONES, Nancy Griselda y TELLO-GARCÍA, María Ascención
}

Universidad Autónoma de Coahuila. Facultad de Enfermería de la Licenciatura en Enfermería "Dr. Santiago Valdés Galindo"

ID $1^{\mathrm{er}}$ Autor: Ma. Guadalupe, Ponce-Contreras / ORC ID: 0000-0003-3174-9682, CVU CONACYT ID: 947777

ID $1^{\text {er }}$ Coautor: Mireya del Carmen González Álvarez / ORC ID: 0000-0002-3061-4439, CVU CONACYT ID: 958313

ID $2^{\text {do }}$ Coautor: Nancy Griselda Pérez-Briones / ORC ID: 0000-0001-6903-4039, CVU CONACYT ID: 370311

ID $3^{\text {er }}$ Coautor: Ma. Ascención, Tello-García / ORC ID: 0000-0002-9057-6497, CVU CONACYT ID: 68385

DOI: $10.35429 / J E S C .2020 .12 .4 .12 .19$

Recibido: 10 de Octubre, 2020; Aceptado 02 de Diciembre, 2020

\section{Resumen}

Los abuelos son hoy pieza fundamental para el funcionamiento del país. Lo eran antes, probablemente lo fueron siempre como referente familiar y social pero los años han reforzado su papel y ampliado notablemente sus responsabilidades (Liñán 2013). Esta investigación pretende describir las actividades del cuidado de los nietos y la calidad de vida de las abuelas. La investigación es de tipo descriptiva, transversal, Muestra; fue de 13 mujeres de 60 años o más que cuidan al menos un nieto y que forman parte del grupo de apoyo del Centro de Salud Girasol, en Saltillo Coahuila. Se utilizaron 4 instrumentos, $1^{\circ}$ una cédula de datos sociodemográficos donde se incluyó la siguiente información: edad, lugar de nacimiento, estado civil, escolaridad, enfermedades que padece, número de hijos, número de nietos, edades de los nietos a los que cuida y con quien vive actualmente. El $2^{\circ}$ describe el cuidado de los nietos, el $3^{\circ}$ mide la calidad de vida y el $4^{\circ}$ mide la percepción de la salud de los adultos mayores. Resultados: El $85.7 \%$ de las adultas mayores no cuenta con un empleo en la actualidad, solamente el 7.1\% de nuestra muestra dispone de un trabajo actualmente. Todas las abuelitas cuidan al menos un nieto.

Adultos, Abuela, Calidad de vida, Nietos

\begin{abstract}
Grandparents are today a fundamental part of the functioning of the country. They were before, they probably always were as a family and social reference, but the years have reinforced their role and notably expanded their responsibilities (Liñán 2013). This research aims to describe the activities of caring for grandchildren and the quality of life of grandmothers. The research is descriptive, transversal, Sample; It was made up of 13 women aged 60 or over who care for at least one grandchild and who are part of the support group of the Sunflower Health Center, in Saltillo Coahuila. 4 instruments were used, 1st a sociodemographic data card where the following information was included: age, place of birth, marital status, schooling, illnesses suffered, number of children, number of grandchildren, ages of the grandchildren they care for and who you currently live with. The 2 nd describes the care of grandchildren, the $3 \mathrm{rd}$ measures the quality of life and the 4th measures the perception of the health of the elderly. Results: $85.7 \%$ of older women do not currently have a job, only $7.1 \%$ of our sample currently have a job. All grannies take care of at least one grandchild.
\end{abstract}

Adults, Grandmother, Quality of life, Grandchildren

Citación: PONCE-CONTRERAS, María Guadalupe, GONZÁLEZ-ÁLVAREZ, Mireya Del Carmen, PÉREZ-BRIONES, Nancy Griselda y TELLO-GARCÍA, María Ascención. Los adultos mayores, el cuidado de los nietos y su calidad de vida. Revista Ciencias de la Educación. 2020. 4-12: 13-20

\footnotetext{
* Correspondencia del Autor (Correo electrónico: mariaponce@uadec.edu.mx)

$\dagger$ Investigador contribuyendo como primer autor.
} 


\section{Introducción}

Los adultos mayores especialmente las mujeres, cumplen con la responsabilidad del cuidado de los nietos debido a la existencia de lazos afectivos y la cultura androcéntrica, donde la mujer funge como la principal responsable y cuidadora natural, lo que las lleva a aceptar voluntariamente la carga de trabajo, debido al compromiso que se tiene hacia los hijos para que ellos puedan continuar con su vida laboral (Liñán, 2018).

Uno de los factores más relevantes por el cual las adultas mayores se hacen cargo de los nietos, viene dado a partir de 1980 cuando las mujeres comienzan a incorporarse a la vida laboral y al aumento de la esperanza de vida en los mayores (Cardo; García; López; Rosello, 2016). Estar al cuidado de los niños requiere mantener una atención constante, implica situaciones de estrés, descuidan su propia salud para cuidar de la de los demás y reduce el tiempo de socialización, lo que impide la relajación y liberar tensiones, por estos motivos, patologías como la hipertensión son mucho más frecuentes en estas mujeres (Noguer, 2018).

La delegación del cuidado de los hijos en otra mujer de la familia (frecuentemente la abuela materna) es una de las estrategias de compatibilización familia-empleo más utilizada. La educación y valores aprendidos por las abuelas provocan que se sientan responsables del permanente cuidado de la familia y lo ven como una forma de seguir sintiéndose útil. La familia delega la responsabilidad a la abuela porque existen lazos afectivos, por comodidad, por no contar con recursos económicos suficientes y además porque no tiene costo (Liñán, 2018). El cuidar a los nietos repercute en la salud de la abuela, lo que trae consigo muchas desventajas del rol de cuidador.

En un estudio publicado por la International Jornual of Developmental and Educational Psychology "las abuelas cuidadoras de sus nietos/as: tareas de cuidado, beneficios y dificultades del rol" reportó como resultado que las mayores desventajas del rol de cuidador de los nietos es el cansancio, sentirse atado y no tener tiempo para ellos, además de que la edad del nieto influyó negativamente en cuanto a no tener tiempo libre (Cardo, et al, 2016).
Dichas desventajas se ven manifestadas en la salud de la adulta mayor originando graves y progresivos desequilibrios tanto somáticos como psicológicos. Algunas de las manifestaciones físicas que suelen presentarse son hipertensión arterial, padecimientos metabólicos, taquicardias, sofocos, dificultad para respirar, mareos, hormigueos, desvanecimientos, cansancio y caídas fortuitas.

Las manifestaciones psicológicas que se presentan son malestar general, disconfort y falta de confort, ansiedad, tristeza, desanimo, falta de apetito, falta de desmotivación, sentimientos de culpa, pensamientos suicidas (Triado, 2019). Un ejemplo claro se puede encontrar en la investigación que lleva por nombre De Hiperfrecuentadora a Abuela Esclava (Gonzales et al 2016) en la cual se menciona el caso de una paciente quien acudió a urgencias 299 veces por diversas manifestaciones clínicas, al realizar la entrevista se obtuvo como resultado que la paciente tenía una acumulación de obligaciones no adecuada para su edad y alguna falta de consideración por parte de sus familiares, lo cual agravaba sus patologías ya diagnosticadas como la hipertensión arterial, sinusitis crónica y síndrome ansioso depresivo.

Algunos de los problemas emocionales más frecuentes que se generan en las abuelas al estar sobrecargadas de actividades son los siguientes: sentimiento de culpa, baja autoestima, falta de concentración, dolores de cabeza, cuadros de tristeza y abatimiento (Alfaro, 2018). Según Acosta (2016) los principales aspectos negativos del cuidado de los nietos es el cansancio, sentirse atado sin tiempo para ellos mismos, se relacionó la línea familiar de proveniencia de estos nietos, ya que se observa una mayor importancia del cuidado en nietos de la línea materna, hecho que viniera a fortalecer la idea de que estos cuidados ayudan a las hijas de estas personas mayores a poder continuar con su vida laboral. Las pacientes suelen negar que están sometidas a estrés por razones familiares o culturales, aunque inconscientemente sabe que tiene demasiadas responsabilidades.

Por su parte la familia ignora la situación consciente o inconscientemente, en ocasiones la abuela está sometida a un estrés constante, lo cual puede ocasionar problemas de salud debido a la gran presión en la que se encuentra las abuelas (Liñán, 2018). 
Aun así, en el estudio de Muñoz y Zapater (2016) los resultados arrojaron que el $36,6 \%$ de las abuelas percibían sobrecarga de trabajo, también se da el caso que el adulto mayor tiene la sensación de realizar una jornada de trabajo a tiempo completo y se sienten forzados en una carga de trabajo de cuidados y educación (López, 2015).

Acosta, Gonzales (2016) en su investigación mencionan algunas de las actividades que se consideran aceptables para los adultos mayores, entre ellas se encuentran las actividades de independencia (ir de compras, lavar trastes, cocinar y barrer), actividades recreativas mentales (ver televisión, jugar juegos de mesa, hacer manualidades, coser y bordar), actividades sociales (ir a fiestas, a la iglesia, platicar con familiares, vecinos o convivir en grupos de adultos mayores) y finalmente actividades de protección de la salud (ir al médico, comer saludablemente, cuidado bucal y tomar siestas).

Por este motivo el objetivo de esta investigación es describir las actividades del cuidado de los nietos y la calidad de vida de las abuelas que forman parte del grupo de apoyo del Centro de Salud Girasol.

\section{Cuerpo Principal}

Literatura Revisada La literatura revisada menciona que el cuidado de los nietos puede afectar en la calidad de vida de las abuelas lo cual puede generan graves estados de salud tanto físicos como psicológicos, por este motivo las enfermedades que ya padecen las adultas mayores se agravan drásticamente como consecuencia de la gran acumulación de obligaciones no adecuada para su edad, una autopercepción mala referente a su salud y una falta de consideración por parte de sus familiares. Las mujeres son las más propensas a asumir este tipo de responsabilidades, lo cual parece ser un deber impuesto por la sociedad.

Las mujeres mayores al tener gran carga de trabajo manifiestan graves y progresivos desequilibrios, tanto somáticos como psicológicos sobre todo aquellas que destinan más horas al cuidado de los nietos. Las abuelas sufren estrés debido al nivel tan alto de presión al que está sometidas constantemente lo cual sobrepasa su capacidad de respuesta.
Otra manifestación importante es el nivel de depresión que sufren las abuelas debido a la gran carga acumulación a la que están sometidas. Una de las principales razones por las cuales las abuelas aceptan cuidar a los nietos es la existencia de vínculos familiares y para ellas es una manera de ayudar a sus hijos para que puedan trabajar lejos de su hogar. Se identificó que las abuelas disfrutan el cuidar a los infantes porque provoca en ellas gran satisfacción y disfrutan de su cercanía. Pero aun así se destaca el hecho de que se sienten atadas y no tienen tiempo para ellas, además del gran cansancio que sufren.

Desde la perspectiva de Pena N (2020) en una investigación realizada en Paraguay, en este artículo el autor analiza las formas en las que se organiza el cuidado de adultos mayores paraguayos con especial foco en áreas rurales y en aquellas familias cuyos hijos han migrado a Argentina. Como insumo principal se analizan entrevistas y observaciones de un trabajo de campo binacional. Se examinan prácticas de cuidados en espacios colectivos y recursos diferenciados a los que acceden las familias transnacionales.

La investigación concluye que, en contextos de aislamiento y escasos apoyos estatales, la participación en familias trasnacionales mejora las condiciones de vida de adultos mayores en Paraguay rural. Además, reflexiona en torno a la necesidad de avanzar hacia modelos conceptuales adecuados que faciliten avanzar en el desarrollo de políticas nacionales y transnacionales que garanticen responsabilidades públicas en el cuidado de adultos mayores

\section{Definición de conceptos}

Calidad de vida: Hace alusión a varios niveles de la generalidad, desde el bienestar social o comunitario hasta ciertos aspectos específicos de carácter individual o grupal. El bienestar físico (como salud, seguridad física), bienestar material (privacidad, alimentos, vivienda, transporte, posesiones), bienestar social (relaciones interpersonales con la familia, las amistades, etcétera), desarrollo y actividad (educación, productividad, contribución) y bienestar emocional (autoestima, estado respecto a los demás, religión) (Ávila, 2013). 
El reflejo de los riesgos y beneficios de nuevos tratamientos, así como del impacto de la enfermedad y de su tratamiento sobre el individuo (Secretaria De Salud) (Instituto Nacional de Ciencias Médicas y Nutrición de Salvador Zubiran).

Cuidado de los nietos: Son actividades realizadas por los abuelos que incluyen acompañar e ir a recoger a sus nietos al colegio y a las actividades extraescolares y fiestas, durante parte de las vacaciones, cuando tienen alguna enfermedad banal, alimentarles, lavarles, acompañarlos en los deberes de colegio, incluso acunarles, substituyendo gran parte del rol de los padres durante las jornadas laborales. (Herrero y Esquirol, 2016)

\section{Metodología}

Diseño:La presente investigación es de tipo descriptiva porque solo se están describiendo las variables, además es una investigación en la que no se manipulan ni se controlan las variables, es exploratorio porque se está conociendo sobre el tema y hay pocas investigaciones sobre el tema. El diseño es transversal porque se centra en analizar la relación de las variables en un momento dado o punto de tiempo y es prospectivo porque se diseña y empieza a realizarse en el presente (Wayne, 2012).

Población: Mujeres de 60 años y más que sean parte del grupo de apoyo en el Centro de Salud Girasol, de Saltillo Coahuila; y además que realicen actividades domésticas y extradomésticas como el cuidado de los nietos. Muestra: 13 mujeres de 60 años o más que cuidan al menos un nieto y que forman parte del grupo de apoyo del Centro de Salud Girasol, en Saltillo Coahuila de Zaragoza. Instrumentos de Medición: Variables sociodemográficas En la presente investigación se utilizó una cédula de datos sociodemográficos donde se incluyó la siguiente información: edad, lugar de nacimiento, estado civil, escolaridad, enfermedades que padece, número de hijos, número de nietos, edades de los nietos a los que cuida y con quien vive actualmente. Se aplicarán tres instrumentos de investigación encargados de describir el cuidado de los nietos la calidad de vida y percepción de la salud de las adultas mayores.
Cuidado de los nietos: Se centra en preguntas referidas al nieto/a que se cuida más intensamente, y a la relación que se mantiene con él, las actividades que lleva a cabo la abuela por el infante. Las tareas de cuidado que se proporcionan se dividen en cinco categorías, de cuidados básicos, cuidados instrumentales, actividades de ocio, disciplina, tareas relacionadas con el estudio. Cuenta con 25 interrogaciones las cuales se responderán con cinco opciones, nunca, pocas veces, algunas veces y muchas veces dependiendo de la frecuencia con la que se realiza cada actividad. Cada una de estas tareas se señalará a partir de un ítem con cuatro posibilidades de respuesta, graduadas desde nunca a muchas veces. Para interpretar los resultados solo se medirá la frecuencia con la que son realizadas las diferentes tareas.

Calidad de vida: Para valorar la intensidad de la calidad de vida que tiene la abuela se aplicó un cuestionario WHOQOLBREF, el cual se divide en cuatro categorías, salud física, Salud psicológica, relaciones sociales y el Ambiente físico. Cuenta con 26 preguntas de opción múltiple donde ellas tomaran en cuenta su modo de vivir, expectativas, placeres y preocupaciones. Dicho instrumento fue diseñado para toda la población en general y proporciona una manera rápida de puntear los perfiles que se desean explorar. Su escala de medición tiene 5 incisos del uno al cinco se contestará dependiendo de la precepción de cada persona, uno se tomará como lo más mínimo o nada y cinco extremadamente o siempre.

El instrumento muestra resultados de las últimas dos semanas. Cuanto mayor sea la puntuación en cada dominio, mejor es el perfil de calidad de vida, de la persona evaluada. Para calcular los resultados se debe realizar las siguientes opciones, confirmar que todas las interrogaciones estén bien contestadas del uno al cinco como se muestra en la escala, se revertirán negativamente tres preguntas la pregunta 3,4 y 26 , si responde 1 cambiara su puntación a 5 , el número 2 se alternara por el 4 , y únicamente el número 3 quedara igual no cambiara, se utilizara el mismo procedimiento si los datos están inversos. Se calculará puntajes por dominio, se sumarán todas las preguntas que pertenezcan a cada apartado y se multiplicara por cuatro los resultados. Realizar la equivalencia del puntaje bruto. 
La información obtenida de la investigación realizada en el centro de Salud Girasol se presenta a continuación en forma de tablas estadísticas, para la presentación de la información y para su fácil interpretación. La información fue introducida en la base de datos de un programa estadístico, ya que permite efectuar análisis estadísticos básicos como avanzados, y permite manejar grandes bancos de datos de manera eficiente. A las variables cualitativas se les midió moda, mediana, media, desviación, rango máximo y rango mínimo. En cuanto a las variables cualitativas, solo se obtuvieron datos de frecuencia y porcentaje.

La forma en que se midieron los instrumentos de evaluación fue la siguiente: la escala que mide las tareas de cuidado de los nietos no cuenta con interpretación, solamente se mide de acuerdo con la frecuencia en porcentaje. En cuanto a la escala de calidad de vida, se tomó como punto de referencia la mediana para decidir que las personas que tuvieran un puntaje menor al de la mediana cuentan con baja calidad de vida y los que cuenten con un puntaje mayor tienen una calidad de vida mayor.

\section{Resultados}

Variables Sociodemográficas: Trabaja actualmente. El $85.7 \%$ de las adultas mayores no cuenta con un empleo en la actualidad, solamente el $7.1 \%$ de nuestra muestra dispone de un trabajo actualmente.

Número de nietos: El $42.9 \%$ de las abuelas cuida solamente a un infante, pero el $14.3 \%$ de las adultas cuidan dos pequeños, siendo el mismo porcentaje de las abuelas que cuidan a tres de sus nietos, y aumentan un poco las cifras ya que el $21.4 \%$ de las adultas se encargan del cuidado de cuatro nietos. El 7.1\% ha cuidado a 30 nietos.

Estado civil: El $42.9 \%$ de las adultas se encuentran casadas, el $35.7 \%$ son viudas y el $13.5 \%$ de las abuelas son solteras. Escolaridad: Los resultados muestran que el $100 \%$ de las adultas mayores abarcaron sus estudios hasta la primaria, se observó que algunas de ellas no lograron concluir con sus estudios cursando solamente con los primeros niveles de primaria. Enfermedades que padecen:
Los resultados nos arrojan las enfermedades que padecen las adultas mayores que cuidan a sus nietos, el $92.1 \%$ de ellas esta diagnosticada con hipertensión arterial, más de la mitad de las abuelas también cuentan diabetes que es el $64.3 \%$ todas sometidas a tratamiento médico, y el $14.2 \%$ padece de dolores articulares en las extremidades inferiores.

Edad: En base a la observación de la edad de las abuelas, se percató que el rango de edad más repetido es de 60 años en tanto que la media de 60 años. En tanto que la media es de 66 años (DE+/-7.5). El máximo de edad fue 88 años y el mínimo fue de 60 años.

Lugar de nacimiento: $\mathrm{El} 42.8 \%$ de los participantes nacieron en saltillo, Coahuila el resto de las encuestadas nacieron en diferentes estados de la republica (7.1\% Michoacán, Ramos Arizpe, San Luis Potosí, Veracruz, Tamaulipas y el Ejido morad)

Edad de los nietos: Las abuelas cuidan a sus nietos en diferentes etapas de su vida algunas desde la infancia hasta la adolescencia, los datos nos muestran las edades que tienen los nietos, el dato mínimo obtenido es de 2 años de edad y el máximo es de 18 años, la media de edad es de 10 años (DE+/-5.6)

Integrantes de familia: El número de integrantes de familia con los que vive la adulta mayor abarca desde 1 integrante hasta 8 personas por domicilio, se observa que el $21.4 \%$ de las abuelas viven con solamente con una persona o dos, los datos disminuyen al ser solamente el $7.1 \%$ aquellas abuelas que viven con más de 5 personas. Según la escala que mide la frecuencia con la que se realizan las tareas de cuidado de los nietos, las actividades se dividen en cinco subgrupos: básicos, instrumentales, estudio, ocio y disciplina.

Entre las tareas referentes a las actividades básicas se encuentran darle de comer al nieto, actividad que es realizada con frecuencia por un $78.60 \%$ de las encuestadas en tanto que solo un $7.10 \%$ nunca lo realizan. Lavarle, cambiarle y peinarle es realizado regularmente por un $64.30 \%$ de las abuelas contra el $21.40 \%$ que no lo realizan. Con estos resultados se puede observar que la mayoría de las abuelas encuestadas realizan con mucha frecuencia dichas actividades consideradas como básicas. 
Si se habla de actividades un poco más complejas, se encuentran las tareas instrumentales. Las que son realizadas con mayor regularidad son: prepararle la comida, $71.40 \%$ de las abuelas afirmaron que lo hacen los siete días de la semana y solo el $7.10 \%$ apuntaron que nunca llevan a cabo esta tarea. El 57.10\% del total de la muestra admitieron ser ellas las encargadas de llevar o traer a su nieto del colegio. En cuanto a lo referente al estudio el $28.60 \%$ nunca ha ayudado con su tarea escolar al nieto, todo lo contrario al $42.90 \%$ que siempre ayuda al nieto. En cuanto a disciplina se observa que las abuelas cuentan con la autoridad de poder regañar a su nieto cuando sea necesario ya que el $64.30 \%$ puede hacerlo seguido pero aun así el $42.90 \%$ de ellas nunca ha dado un golpe al nieto ya que no lo creen necesario (ver tabla 1).

\section{Conclusiones}

La escala de calidad de vida arrojó que solamente el $14.3 \%$ de las abuelas considera tener una muy buena calidad de vida y el $85.3 \%$ de las abuelas encuestadas no se encuentran totalmente satisfecha con el nivel de vida en el que se encuentran. En cuanto al tema de la salud, se observa que son escasas las abuelas que se encuentran muy satisfechas con su estado de salud ya que solamente el $21.4 \%$ está conforme. Igualmente, el $21.4 \%$ esta insatisfechas con su estado de salud. Solo el $21.4 \%$ del total de las encuestadas afirman disfrutar la vida y solo el $28.6 \%$ de ellas le encuentran el sentido a su vida.

Se observa que las adultas creen que su calidad de vida va de lo normal a lo extremadamente bien, muy pocas consideran estar inconformes en la forma en la que viven pero a pesar de eso se nota un considerable aumento ya que ninguna de ellas cuenta con los recursos económicos suficientes para solventar sus necesidades económicas básicas, se percató que no están muy conformes con el ambiente físico a su alrededor ya que este no es saludable para ellas, solamente el $14.3 \%$ es capaz de aceptar su apariencia física, el 50\% necesita de un tratamiento médico para funcionar en su vida diaria y señalan que tiene la capacidad de desplazarse solas de un lugar a otro sin ninguna ayuda. Se puede observar que solo el $7.1 \%$ de las abuelas tienen la oportunidad de realizar actividades de ocio frecuentemente y el $14.3 \%$ nunca tienen tiempo para realizarla.
Siguiendo con la calidad de vida, el 42.9\% de las adultas mayores están insatisfechas con su calidad de sueño, solamente el $14.3 \%$ de las abuelas no cuenta con dificultades a la hora de dormir. La mayoría de las adultas se encuentran complacidas con los servicios sanitarios y de transporte público en su zona, el $28.6 \%$ están conformes con las condiciones del lugar donde viven. En las relaciones personales y al recibir apoyo de amigas se muestra demasiado desinterés ya que solamente el $7.1 \%$ se encuentra satisfecha con el apoyo que recibe de parte de las amistades con las que cuenta, en las relaciones personales el $21.4 \%$ se siente muy contento con los vínculos interpersonales.

Para medir el nivel de calidad de vida en el que se encontraban las abuelas encuestadas, se obtuvieron los resultados del instrumento en cuanto a las 4 dimensiones (salud física, salud psicológica, relaciones personales y entorno) mostrándose una considerable disminución en sus relaciones interpersonales. La mediana es de 320 , así que la población que cuenta con un puntaje mayor se considera que tienen un nivel de calidad de vida bueno y aquellas encuestadas que obtuvieron un puntaje menor de la mediana, cuentan con un nivel de calidad de vida bajo.

En cuanto a la Calidad de vida, el $61.5 \%$ de la población tiene baja calidad de vida en comparación con el $38.5 \%$ que cuentan con mayor calidad de vida. $\mathrm{Si}$ relacionamos la calidad de vida con la cantidad de nietos a los que cuida y la edad del nieto mayor se observan variaciones ya que fueron 6 abuelas de las 13 que conformaron la muestra quienes obtuvieron puntajes menores a 320. El puntaje mínimo fue de 236 perteneciente a una abuela que ha cuidado a sus 30 nietos y actualmente la edad del nieto mayor que cuida es de 8 años. Tomando en cuenta los puntajes de las 6 abuelas con baja calidad de vida, las edades de los nietos varían mucho, ya que el dato mínimo es de 7 años y el dato mayor es de 18 años, así como la cantidad de nietos a los que cuida el dato menor fue de 1 y el dato mayor es de 30 .

\section{Referencias}

Acosta, C.\& González, A.. (julio-diciembre 2016). Actividades de la vida diaria en adultos mayores: la experiencia de dos grupos focales. Psicología y Salud, 19, 289-293. 
Alfaro L.M., Téllez-Rojo, (2018) "Condiciones de salud y estado funcional de los adultos mayores en México", en Salud Pública de México, vol. 55, suplemento 2, México, 2018, pp. 323-331.

Alfonso, L., Soto, D., \& Santos, N. (2019). Calidad de vida y apoyo social percibido en adultos mayores. Revista Ciencias Médicas de Pinar del Río, 20(1). Recuperado de https://www.medigraphic.com/pdfs/pinar/rcm2 016/rcm1611.pdf

Ávila S. (2013). Las abuelas cuidadoras opiniones y perspectivas de un grupo de personas mayores. 2011, de Universidad de Salamanca Sitio web: http://gredos.usal.es/xmlui/bitstream/handle/10 366/101363/TFM_EstudiosInterdisciplinaresGe nero_SoldevilaGarnica_M.pdf?sequence $=1$

Cardo; García; López; Rosello, (2016).Cabrera, J.,Martinez,L.,Robles, M.\&Ramos,B.. (julio diciembre 2015). Perfil sociodemográfico, carga percibida y calidad de vida en abuelos cuidadores de niños con discapacidad. Psicología y Salud, 25, 169-180.

Consejo Nacional de Población. Proyecciones de la Población de México 2010-2050. México, CONAPO, 2013.

Encuesta Nacional sobre la Dinámica de las Relaciones en los Hogares, 2013. Base de datos. México, INEGI, 2012.

Encuesta Nacional de Ingresos y Gastos de los Hogares, 2012. Base de datos. México, INEGI, 2013.

Herrero y Esquirol. (2016). Abuelos cuidadores: solidaridad intergeneracional en el contexto de la precariedad e inestabilidad laboral.. 2016, de Universidad de Cádiz Sitio web: http://hdl.handle.net/10498/16813

Gonzales A, Rosillo, D.,Salazar, R.,Raygal, P.,Lazaro,R.,\&Cánovas, J.,. (2016). De hiperfrecuentadora a abuela esclava. Estudio de un caso de sobrecarga del cuidador desde el Síndrome de la Abuela Esclava de Guijarro Morales. 2011, de Enfermería global Sitio web: http://scielo.isciii.es/scielo.php?pid=S169561412011000100004\&script=sci_arttext
Guijarro, A. (2014). El síndrome de la abuela esclava. España: Universitario (Granada).

Liñan, M.,\&Jara, E.. (2018). Principales efectos en la salud física y emocional del adulto mayor, producido por el síndrome del abuelo/a esclavo/a comunidad "Virgen del milagro". 2015, de Universidad de Cuenca Sitio web: http://dspace.ucuenca.edu.ec/bitstream/1234567 89/23055/1/TESIS.DOC.pdf

Instituto Nacional de Estadística y Geografía. Encuesta Nacional de Ingresos y Gastos de los Hogares (ENIGH) 2019. Base de datos.

Instituto Nacional de Estadística y Geografía (INEGI). Estadísticas de mortalidad, 2019. Consulta interactiva de datos. México, INEGI, 2012.

López, J., Domínguez, R. and Delgado, M. (2015). Percepción de los abuelos de la relación con sus nietos: valores, estilos educativos, fortalezas y bienestar. Doctorado. Universidad Complutense de Madrid.

Muñoz y Zapata. M. (2016), Manrique-Espinoza B., Salinas-Rodríguez A., Moreno-Tamayo K.M., Acosta-Castillo I., Sosa-Ortiz A.L., Gutiérrez. "Calidad de vida de los adultos mayores"España: Universitario (Granada)

Murgieri, M.. (2019). Escala de calidad de vida. 2016, de The World Health Organization Quality of Life Sitio web: http://www.juntadeandalucia.es/servicioandaluz desalud/contenidos/publicaciones/Datos/426/pd f/Anexo_3-1_WHOQOL-BREF.pdf

Noguer-Presbiterian. (2018). Abuelos que crían a sus nietos. 2017, de New York - Presbyterian Hospital Sitio web: http://www.cornellcares.org/pdf/handouts/rct_g randparents_sp.pdf

Organización de las Naciones Unidas (ONU). Departamento de Asuntos Económicos y Sociales, División de Población (2019). Perspectivas Mundiales Demográficas: Revisión 2018. 
OMS, "Envejecer bien, una prioridad mundial", Sitio web: http://www.who.int/ageing/es/, 2019. Pena, N. (2020). El cuidado de adultos mayores en Paraguay: estrategias colectivas y circulación de cuidados en familias transnacionales. Revista Latinoamericana De Población, 14(27), 5-40. https://doi.org/10.31406/relap2020.v14.i2.n27.1 Secretaría de Salud (SSA). Egresos Hospitalarios 2012. Base de datos. México, SSA.

Secretaría de Desarrollo Social (Sedesol), Instituto Nacional de las Personas Adultas Mayores (INAPAM), Programa Anual de Trabajo 2014.

Triado,C.,Villar,F.,Solé,C.,Celdrán,M.,Pinazo,S .,Conde,L.,\&Rodriguez,J.,.,. (2019). Las abuelas/os cuidadores de sus nietos/as: Tareas de cuidado, beneficios y dificultades del rol. Revista de Psicología, 4, 455-464.

Wayne, D.. (2012). Bioestadística. Ciudad de México: Limusa. 